\title{
A Novel Probabilistic Linear Subspace Approach for Face Applications
}

\author{
Ying Ying and Han Wang \\ School of Electrical and Electronic Engineering, \\ Nanyang Technological University, \\ 50 Nanyang Avenue, Singapore, 639798 \\ \{yi0002ng, hw\}@ntu.edu.sg
}

\begin{abstract}
Over the past several decades, pattern classification based on subspace methodology is one of the most attractive research topics in the field of computer vision. In this paper, a novel probabilistic linear subspace approach is proposed, which utilizes hybrid way to capture multidimensional data extracting maximum discriminative information and circumventing small eigenvalues by minimizing statistical dependence between components. During features extraction process, local region is emphasized for crucial patterns representation, and also statistic technique is used to regularize these unreliable information for both reducing computational cost and maintaining accuracy purposes. Our approach is validated with a high degree of accuracy with various face applications using challenging databases containing different variations.
\end{abstract}

Keywords: probabilistic analysis, linear subspace, face application.

\section{Introduction}

The ultimate goal of pattern recognition is to discriminate the class of observed objects with the minimum misclassification rate. Thus, in the discriminating process, a pattern recognition system intrinsically utilizes low dimensionality to represent the input data. Subspace analysis is a powerful tool of seeking low-dimensional manifolds which models continuous variations in patterns, and new image can be embedded into these manifolds for classification. Among the numerous techniques published in the past few years, ways directly solves the classification and clustering problems, such as sparse representation [1] and subspace arrangements [2]. Another category emphasizes on feature extraction, like Laplacian Eigenmaps (LE) [3], locality preserving projections (LPP) [4], and marginal Fisher analysis (MFA) [5. The superiority of subspace method can be concluded as aiming at reducing the computational complexity of the classification with minimum loss of discriminative information [6]. This can be done by maximizing the information carried by the data in the extracted low-dimensional subspace, and as evidenced by the fact that vast majority of the proposed approaches are based on "most discriminative" criteria which extract maximum discriminative information in the form of reduced low-dimensional space from 
large scale, such as LE, LPP, and MFA. While the other objectives are to circumvent the over-fitting problem of the classification and enhance the accuracy and robustness. Curse of dimensionality, small sample size, or noise removal effect are some problematic and harmful situations for robust classification. One way is to regularize these unreliable statistic or remove corresponding dimensions. Although various regularization techniques are proposed, they are supposed to be applied before dimensionality reduction because regularization in classification stage cannot recover the improperly removed dimensions in dimensionality stage. Based on the understanding of the roles of subspace method, we can find most top performers of the state-of-art subspace-based approaches adopt either various regularized discriminative analysis or two-stage approaches to realize superiorities and also boost the classification accuracy.

According the principles behind the subspace analysis, here, we propose a novel probabilistic approach for pattern classification. Basically, it adopts multiple linear subspace methods to capture multi-dimensional data which extracts maximum discriminative information and circumventing small eigenvalues by minimizing the statistical dependence between components. During the features extraction process, local region is emphasized for crucial patterns representation, and also statistic technique is used to regularize these unreliable information for both reducing computational cost and maintaining accuracy purposes. Our discussion in this paper is limited on face applications which involves two- and multi-class classification problems, and proposed approach can be also developed for other general pattern recognition tasks.

The remainder of this paper is structured as follows: Section 2 analyzes the proposed probabilistic linear subspace approach. Performances of different face applications and conclusion will be presented in section 3 and 4, respectively.

\section{Probabilistic Linear Subspace Approach}

As one of the most popular subspace methods, PCA yields the projection directions that maximize the data structure information in the principal space across all classes, and hence is optimal for data reconstruction. Although PCAbased classification approaches reduce computational complexity by capturing low-dimensional data, the dimensionality utilized is chosen according to the different targets which means the performance utilizing extracted feature cannot be guaranteed in case of the data with large deviation from training samples. Unfortunately, unwanted variations like lighting, facial expression, or other reality factors always exist. Some work suggests to discard several most significant principal components and better clustering of projected samples can be achieved. Yet, it is unlikely that these principal components correspond solely to variations, as a consequence, information that is useful for discrimination may also lose. On the other hand, PCA-feature only analyzes the first- and second-order statistics information capturing amplitude spectrum of images, since PCA seeks directions that are most independent from each other. High-order statistics containing phase spectrum hold important information which can be transformed be 
variation immunity. Thus, these information should be well modeled for boosting the classification accuracy and robustness.

Classification is to assign a given pattern to one of the categories. The minimum probability of misclassification is achieved by assigning pattern to class that has maximum probability after pattern has been observed, thus the Bayes theory can be utilized to evaluate the discriminant function,

$$
f_{i}(x)=\ln P\left(x \mid C_{i}\right)+a_{i}
$$

where $x$ and $a_{i}$ represent the input and the threshold for class $i$, respectively. For further analysis, data usually is modeled by an analytical form, such like multivariate Gaussian distribution. As the most natural distribution, it is an appropriate second-order statistics model for many situations [6]. After eigendecomposition, Eq. 11 is simplified as,

$$
\begin{aligned}
f_{i}(x) & =-\frac{1}{2}\left(x-\bar{X}_{i}\right)^{T} \Sigma_{i}^{-1}\left(x-\bar{X}_{i}\right)+b_{i} \\
& =-\frac{1}{2} \sum_{j} \frac{\left(w_{j}-\bar{W}_{j}\right)^{2}}{\lambda_{j}}+b_{i}
\end{aligned}
$$

where $\bar{X}_{i}$ and $\Sigma_{i}$ are the mean and covariance distribution, and $w_{j}$ and $\bar{W}_{j}$ are the projections of $x$ and $\bar{X}_{i}$ on the orthonormal eigenvectors corresponding to the eigenvalue $\lambda_{j}$ of $\Sigma_{i}$.

Moghaddam [7] and Jiang [8] suggested to separate the discriminant function into two parts and replace small eigenvalues by a constant,

$$
f_{i}(x)=-\frac{1}{2}\left[\sum_{j=1}^{m} \frac{\left(w_{j}-\bar{W}_{j}\right)^{2}}{\lambda_{j}}+\sum_{j=m+1}^{n} \frac{\left(w_{j}-\bar{W}_{j}\right)^{2}}{\rho}\right]+b_{i}
$$

where $\rho$ is computed as a fixed percentage of corresponding eigenvalues or an upper bound of small eigenvalues. All these adding a constant to all eigenvalues or replacing the unreliable eigenvalues by a constant are reported with better classification performances. These PCA variants still adopt single model to extract discriminate feature and decreased large deviation effect caused by small eigenvalues. However these "unreliable" small eigenvalues actually do contain high-order statistic information which contributes for classification and cannot be simply replaced by a constant instead of completely modeling. One more issue is all these modeling is based on Gaussian distribution assumption, which can not completely describe the reality cases. Inspired from it, we propose our subspace-based two-stage model.

\subsection{Proposed Approach}

Considering the outstanding performance for both dimensionality reduction and discriminative information representation, PCA feature is competent for modeling the first- and second-order statistics. The rest small eigenvalues, as the 
second part decomposed in Eq. 3. can be described by independent component analysis (ICA) 9]. Although ICA is a generalization of linear subspace approach, unlike PCA, it searches for a transformation that minimizes the statistical dependence between components, and thus provides a good representation of data by virtue of exploiting the entire data space. However, ICA loses merit when dealing Gaussian distributed data 10 while showing the superiority in encoding non-Gaussian distribution. As the reasons stated for building up multivariate Gaussian distribution, the existence of non-Gaussian distribution cannot be ignored, such as geometrical variation, which here can be compensated by ICA.

Our strategy is to use PCA to isolate low-order statistical information modeling Gaussian distribution, simultaneously, ICA is applied to represent high-order data which is more superior to model non-Gaussian distribution. Thus, each reconstructed image $\hat{x}$ can be written as,

$$
\begin{aligned}
\hat{x} & =\left(\Phi_{m} \Phi_{n}\right) *\left(\Phi_{m} \Phi_{n}\right)^{T} * x \\
& =\Phi_{m} \Phi_{m}^{T} * x+\Phi_{n} \Phi_{n}^{T} * x \\
& =\Phi_{m} W_{m}+U_{n}\left(W_{i n v t}^{T}\right)^{-1} \Phi_{n}^{T} * x \\
& =\Phi_{m} W_{m}+U_{n}\left(\Phi_{n} W_{i n v t}^{-1}\right)^{T} * x \\
& =\Phi_{m} W_{m}+U_{n} B_{n}
\end{aligned}
$$

where $\Phi_{m}$ and $\Phi_{n}$ are the first $m$ and residual $n$ principle components in the eigenvector matrix $\Phi$, respectively; $W_{m}$ represents the projection in the PCA space; $U_{n}$ and $B_{n}$ denote the independent basis image and coefficient in the residual ICA space, respectively.

Generally, subspace methods encode the gray scale correlation among every pixel position statistically, and any variation can cause severe changes of information representation. However, pattern recognition based on local regions are reported having a demonstrably superior performance to those which exploit global information [1011/2]. Firstly, as local region exhibiting less statistical complexity, method using local space will be more robust to illumination change. Also, local pattern might vary less under pose changes than global one. Lastly, local feature is more robust against partial occlusions since local region recognition is little affected. So Eq. 4 can be further rewritten according to the specific subregions defined containing important and meaningful local features,

$$
\begin{aligned}
\hat{x_{s}} & =\left(\Phi_{s}\right)_{m}\left(\Phi_{s}\right)_{m}^{T} * x_{s}+\left(\Phi_{s}\right)_{n}\left(\Phi_{s}\right)_{n}^{T} * x_{s} \\
& =\left(\Phi_{s}\right)_{m}\left(W_{s}\right)_{m}+\left(U_{s}\right)_{n}\left(B_{s}\right)_{n}
\end{aligned}
$$

Having derived the principal and independent components, each local component is projected onto PCA and ICA spaces, respectively. Thus the statistic analysis is applied to regularize PCA projection weight $W_{m}$ and ICA coefficient $B_{n}$. Because data usually has divergent and complicated distributions in reality case, instead of a single multivariate Gaussian model, a weighted mixture of multivariate Gaussian (GMM) distribution is adopted here to increase modeling precision 
and reduce misclassification rate. The likelihood probability of the $j$ th local region $x_{s}^{j}$ can be written as,

$$
\begin{aligned}
P\left(x_{s}^{j} \mid C_{i}\right) & =\frac{\exp \left[-\frac{1}{2}\left(x_{s}^{j}-\bar{X}_{s}^{j}\right)^{T}\left(\Sigma_{s}^{j}\right)^{-1}\left(x_{s}^{j}-\bar{X}_{s}^{j}\right)\right]}{(2 \pi)^{L / 2}\left|\Sigma_{s}^{j}\right|^{1 / 2}} \\
& =\frac{\exp \left[-\frac{1}{2} d\left(x_{s}^{j}\right)\right]}{(2 \pi)^{L / 2}\left|\Sigma_{s}^{j}\right|^{1 / 2}} \\
& =\frac{\exp \left\{-\frac{1}{2} d\left[\left(x_{s}\right)_{P C A}^{j}\right]-\frac{1}{2} d\left[\left(x_{s}\right)_{I C A}^{j}\right]\right\}}{(2 \pi)^{(m+n) / 2}\left|\left(\Sigma_{s}\right)_{m}^{j}\left(\Sigma_{s}\right)_{n}^{j}\right|^{1 / 2}} \\
& =P\left(\left(x_{s}\right)_{P C A}^{j} \mid C_{i}\right) * P\left(\left(x_{s}\right)_{I C A}^{j} \mid C_{i}\right) \\
& =\sum_{k=1}^{a^{j}} P\left[\left(W_{s}\right)_{k}^{j} \mid \Omega_{k}^{j}\right] * \sum_{l=1}^{b^{j}} P\left[\left(B_{s}\right)_{l}^{j} \mid \Gamma_{l}^{j}\right]
\end{aligned}
$$

where $a^{j}$ and $b^{j}$ are the GMM cluster numbers for PCA and ICA spaces of class $i$, where $\sum a^{j}=1$ and $\sum b^{j}=1 ; d\left(x^{j}\right)$ is the corresponding Mahalanobis distance; $\Omega_{k}^{j}=\left(\pi_{k}^{j}, \mu_{k}^{j}, \Phi_{k}^{j}\right)$ and $\Gamma_{l}^{j}=\left(\varphi_{l}^{j}, \nu_{l}^{j}, \Psi_{l}^{j}\right)$ are the $k$ th and $l$ th Gaussian parameter sets containing likelihood probability weights $\pi_{k}^{j}$ and $\varphi_{l}^{j}$, mean vectors $\mu_{k}^{j}$ and $\nu_{l}^{j}$, covariance matrixes $\Phi_{k}^{j}$ and $\Psi_{l}^{j}$ in PCA and ICA spaces, respectively. For estimating parameter sets $\Omega_{k}^{j}$ and $\Gamma_{l}^{j}$, the initial values of mean $\mu_{k}^{j}$ and $\nu_{l}^{j}$, and covariance $\Phi_{k}^{j}$ and $\Psi_{l}^{j}$ of cluster $j$ in class $i$ can be calculated by being partitioned by modified k-means clustering, which uses the likelihood probability of multivariate Gaussian model as the measure of the nearest neighbor rule. The corresponding initial weights $a^{j}$ and $b^{j}$ are defined as the ratio of subspace data number in cluster $j$ to the total training number. Then the expectation maximization (EM) algorithm iteratively optimizes $\Omega_{k}^{j}$ and $\Gamma_{l}^{j}$ to local maximum in the total likelihood of the training set. During the integration procedure [1], likelihood probability of the entire image $x$ can be expressed as,

$$
P\left(x \mid C_{i}\right)=\prod_{j=1}^{Q} P\left(x_{s}^{j} \mid C_{i}\right)
$$

where $Q$ is the subregion number. Therefore, the discriminant function Eq 1 becomes,

$$
f_{i}(x)=\ln \left[\prod_{j=1}^{Q} P\left(x_{s}^{j} \mid C_{i}\right)\right]+a_{i}=\sum_{j=1}^{Q} \ln \left[P\left(x_{s}^{j} \mid C_{i}\right)\right]+a_{i}
$$

and $x$ is classified to the class with maximum probability.

The outline of the proposed probabilistic subspace approach for both training and testing procedures is shown in Fig. 1. 
(a) Training Process

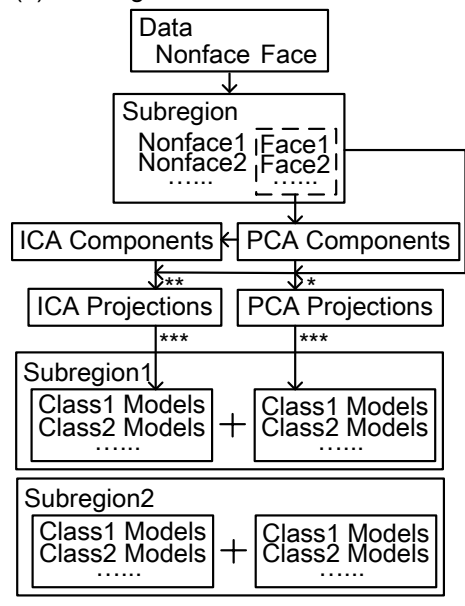

(b) Testing Process

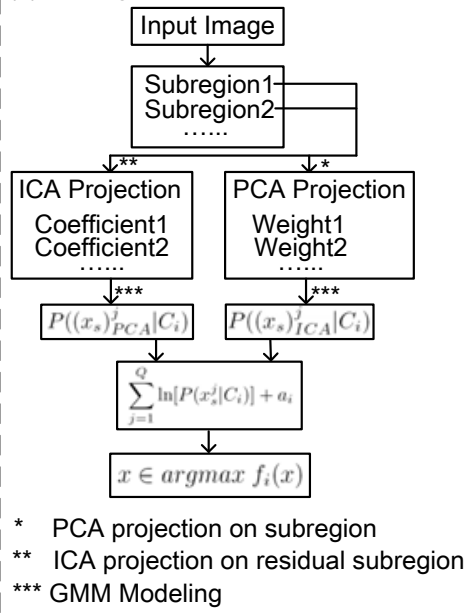

Fig. 1. Flowcharts of proposed approach: (a) training, and (b)testing process

\section{Experiment}

\subsection{Multi-view Face Detection}

Similar to the work [13, the experiment platform adopted here comprises preprocessing, detection, and postprocessing modules. After geometric normalization and lighting correction utilized in the preprocessing, three view-based face detectors are trained by the proposed approach using canonical facial data, which continuously cover the whole out-of-plane rotation. Postprocessing including grouping, averaging, and filtering is used to solve multi-resolution issue, and gives unambiguous location and scale of face without information loss.

Experiment data includes FacePix [1415], PIE, Pointing04 [16], and CMU profile [1] datasets as face images; Caltech background dataset, Caltech and Pasadena Entrances 2000 dataset, Caltech Houses dataset and Fifteen Scene Categories dataset as nonface images. FacePix provides multi-view faces with continuous pose changing, PIE and Pointing04 have discrete pose changing faces, and CMU datasets contain faces with unestimated poses under complex background. Canonical images, subregions, PCA reconstructed subregions, and residual subregions defined for different face views are illustrated in Fig. 2. It can be seen that reconstructed subregions are similar to their low-pass filtered equivalents and the residual ones which are characterized by high-frequency components are less sensitive to illumination variations, which is equivalent to the low- and high-order statistics separation.

The proposed subspace model, designated as "PCA+ICA", is compared with other two approaches, namely "PCA" and "ICA", in which PCA and ICA are employed solely to model data respectively. The current "PCA+ICA" model 


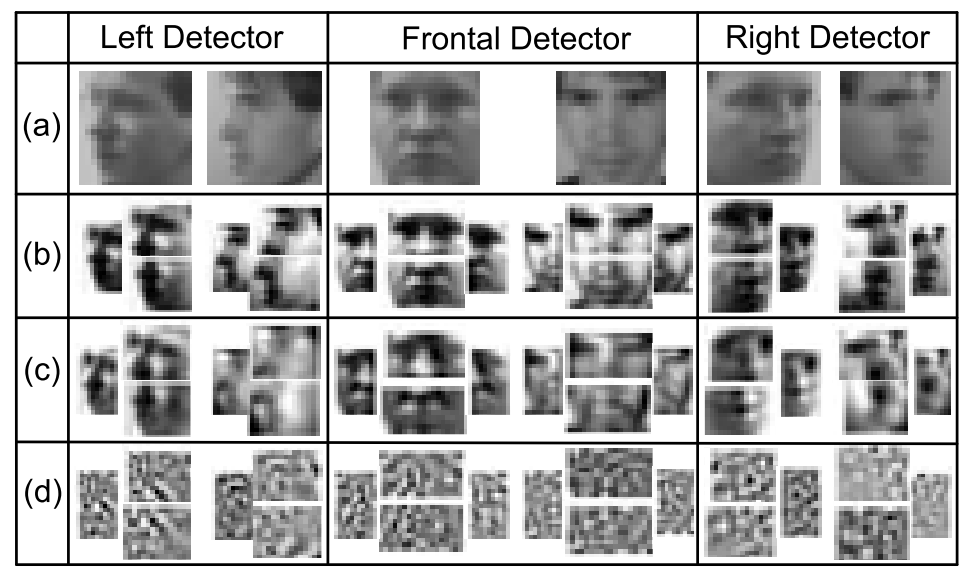

Fig. 2. (a) Original images; (b) subregions; (c) PCA reconstructed subregions; and (d) residual subregions

yields the best performance which demonstrates information distributed in the low- and high-frequency components are appropriately preserved by PCA and ICA, respectively, see Fig. 3a, Another experiment is conducted between current local subspace face detector, designated as "Local+PCA+ICA", and the "Global+PCA+ICA" detector in which the input is the entire patch. Fig. 3b plots the corresponding ROC curves, in which the "Local+PCA+ICA" yields better result than the global one.

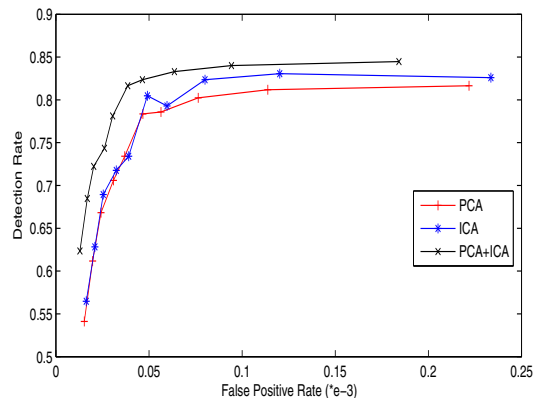

(a) PCA vs. ICA vs. PCA+ICA

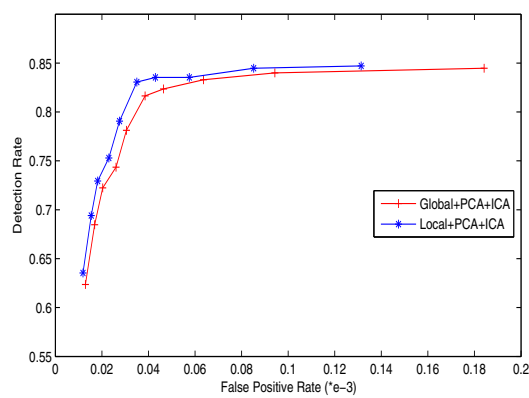

(b) Global vs. Local

Fig. 3. ROC for multi-view face detection based on different approaches

Besides the evaluations conducted for showing the superiority of the proposed approach, quantitative experiment also is done and comparison with other outstanding methods are summarized in Table 1. Results using FacePix, PIE, 
Pointing'04, and CMU profile data averagely achieve around $90 \%$ detection rates. Especially for CMU profile, compared to other methods, the proposed method obtains an acceptable detection rate with reasonable false alarm. Meanwhile, the pose of detected face is coarsely estimated according to the detector from which the patch survives. Some typical examples detected by the proposed method are shown in Fig. 4 .

Table 1. Performance of multi-view face detection and comparison between proposed method and others

\begin{tabular}{|c|c|c|c|}
\hline Database & Method & Detection Rate & False Positive Number \\
\hline FacePix & our & $94.13 \%$ & 281 \\
\hline PIE & our & $88.76 \%$ & 1348 \\
\hline Pointing'04 & our & $91.52 \%$ & 537 \\
\hline \multirow{4}{*}{ CMU profile } & Schneiderman [1] & $92.7 \%$ & 700 \\
\cline { 2 - 4 } & Jones [17] & $83.1 \%$ & 700 \\
\cline { 2 - 4 } & Wu [18] & $91.3 \%$ & 415 \\
\cline { 2 - 4 } & our & $86.17 \%$ & 715 \\
\hline
\end{tabular}

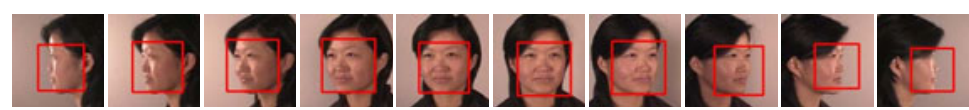

(a) FacePix dataset

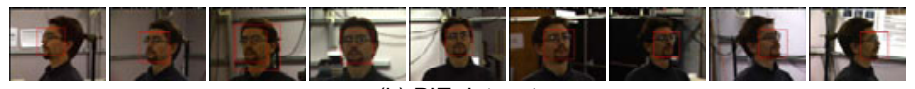

(b) PIE dataset

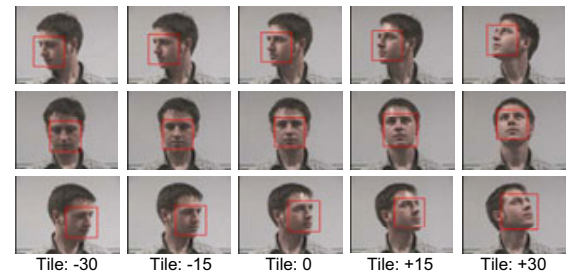

(c) Pointing'04 dataset

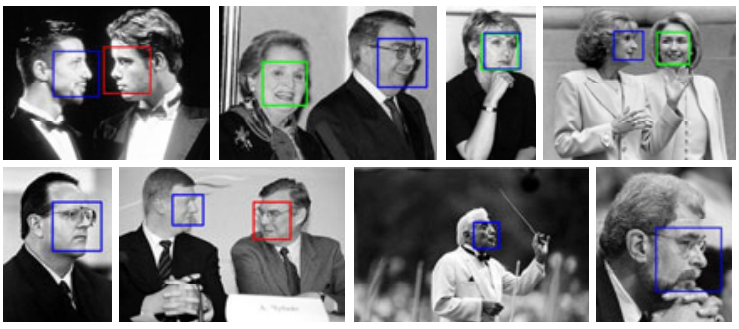

(d) CMU profile dataset

Fig. 4. Results for multi-view face detection application tested on different databases; for $\mathrm{CMU}$ profile dataset, red, green, and blue rectangles present results detected by left, frontal, and right detectors, respectively 


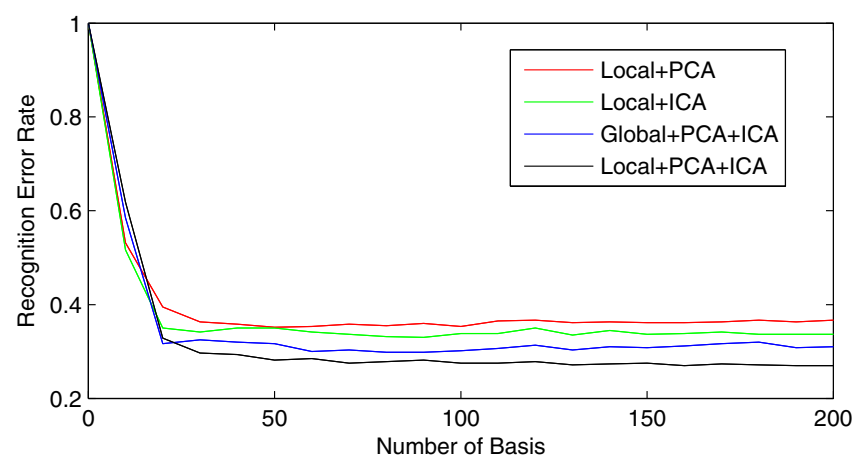

Fig. 5. Top recognition error rate for the number of selected basis components

\subsection{Face Recognition}

The proposed method is applied for face recognition and satisfied performance is achieved. The evaluation performance is conducted based on AR database [19], including frontal view faces with different facial expressions, illumination conditions, and partial occlusions. For each subject, 20 images are randomly chosen and treated as gallery, and the left 6 images are for testing. "Local+PCA+ICA", "Global+PCA+ICA", "Local+PCA", and "Local+ICA" curves are shown in Fig. 5. which clearly prove that our model has advantage over other ones.

The two- and multi-class pattern classification applications demonstrated above consistently show the classification ability of our probabilistic subspace approach. Based on the PCA work, the ICA model representing the residual high-order statistic information reveals the ability of describing non-Gaussian distribution. Especially with the local component emphasizing, the robustness and accuracy are both boosted since partial occlusion, illumination, geometry variations are alleviated to different extent.

\section{Conclusion}

This paper proposes a probabilistic two-stage linear subspace method for pattern classification. Besides the principal dimension statistics extracted by PCA, highorder information is analyzed by ICA model by projecting the corresponding coefficient to its feature space. Not only this, non-Gaussian distribution modeled by ICA feature shows the same importance as Gaussian assumption for pattern recognition. On the other hand, feature within subregions shows strong robustness against variations, e.g. lighting, geometry, and occlusion, since features are selectively emphasized. The weighted GMM probabilistic model also makes data representation more completely. ICA model, local feature, and probabilistic analysis all contribute to classification accuracy boosting, while being exempted from ICA-reconstruction helps to keep from the reconstruction cost. It can be concluded from experiments that the performances of our method in 
both face detection and recognition experiments are satisfactory with images characterized by a wide variety.

\section{References}

1. Wright, J., Yang, A.Y., Ganesh, A., Sastry, S.S., Ma, Y.: Robust Face Recognition Via Sparse Representation. TPAMI 31, 210-227 (2009)

2. Vidal, R., Ma, Y., Sastry, S.: Generalized Principal Component Analysis (GPCA). TPAMI 27, 1945-1959 (2005)

3. Belkin, M., Niyogi, P.: Laplacian Eigenmaps for Dimensionality Reduction and Data Representation. Neural Computation 15, 1373-1396 (2003)

4. He, X., Yan, S., Hu, Y., Niyogi, P., Zhang, H.J.: Face Recognition Using Faces. TPAMI 27, 328-340 (2005)

5. Yan, S., Xu, D., Zhang, B., Yang, Q., Zhang, H., Liu, S.: Graph Embedding and Extensions: A General Framework for Dimensionality Reduction. TPAMI 29, 40-51 (2007)

6. Jiang, X.D.: Linear Subspace Learning-Based Dimensionality Reduction. Signal Processing Magazine 28, 16 (2011)

7. Moghaddam, B.: Principal Manifolds and Probabilistic Subspace for Visual Recognition. TPAMI 24, 780-788 (2002)

8. Jiang, X.D., Mandal, B., Kot, A.C.: Enhanced Maximum Likelihood Face Recognition. Electronic Letter 42, 1089-1090 (2006)

9. Bartlett, M.S., Movellan, J.R., Sejnowski, T.J.: Face Recognition by Independent Component Analysis. Neural Networks 13, 1450-1464 (2002)

10. Kim, T.K., Kim, H., Hwang, W., Kittler, J.: Independent Component Analysis in a Local Facial Residue Space for Face Recognition. Pattern Recognition 37, 1873-1885 (2004)

11. Schneiderman, H., Kanade, T.: A Statistical Method for 3D Object Detection Applied to Faces and Cars. In: IEEE International Conference on Computer Vision and Pattern Recognition, pp. 746-751 (2000)

12. Heisele, B., Serre, T., Poggio, T.: A Component-Based Framework for Face Detection and Identification. IJCV 74, 167-181 (2007)

13. Ying, Y., Wang, H., Xu, J.: An Automatic System for Multi-View Face Detection and Pose Estimation. In: IEEE Internation Conference on Control, Automation, Robotics and Vision, pp. 1101-1108 (2010)

14. Black, J., Gargesha, M., Kahol, K., Kuchi, P., Panchanathan, S.: A Framework for Performance Evaluation of Face Recognition Algorithms. In: Internet Multimedia Systems II ITCOM (2002)

15. Little, G., Krishna, S., Black, J., Panchanathan, S.: A Methodology for Evaluating Robustness of Face Recognition Algorithms with Respect to Changes in Pose and Illumination Angle. In: ICASSP (2005)

16. Gourier, N., Hall, D., Crowley, J.L.: Estimating Face Orientation from Robust Detection of Salient Facial Features. In: International Workshop on Visual Observation of Deictic Gestures (2004)

17. Jones, M., Viola, P.: Fast Mulit-View Face Detection. Technical Report TR2003-96, Mitsubishi Electric Research Labs (2004)

18. Wu, B., Ai, H.Z., Huang, C., Lao, S.H.: Fast Rotation Invariant Multi-View Face Detection Based on Real Adaboost. In: IEEE 6th International Conference on Automatic Face and Gesture Recognition, pp. 79-84 (2004)

19. Martinez, A.M., Benavente, R.: The AR face database. CVC Tech. Report \#24 (1998) 